\title{
Erratum: Large anomalous Nernst coefficient in an oxide skyrmion crystal Chern insulator [Phys. Rev. B 98, 205125 (2018)]
}

Yo Pierre Mizuta, Hikaru Sawahata, and Fumiyuki Ishii

(Received 30 June 2020; published 16 July 2020)

DOI: 10.1103/PhysRevB.102.039903

In Fig. 5(c) of the original article, incorrectness of thermoelectric conductivities $\alpha_{x x}$ and $\alpha_{x y}$ by a constant factor has been noticed. In this Erratum is the corrected version of Fig. 5. Specifically, the correction made is nothing but multiplication of the originally plotted values by a factor of 600 (note the revision of unit for the horizontal axis).

This correction does not affect any other part of the original article.

(a)

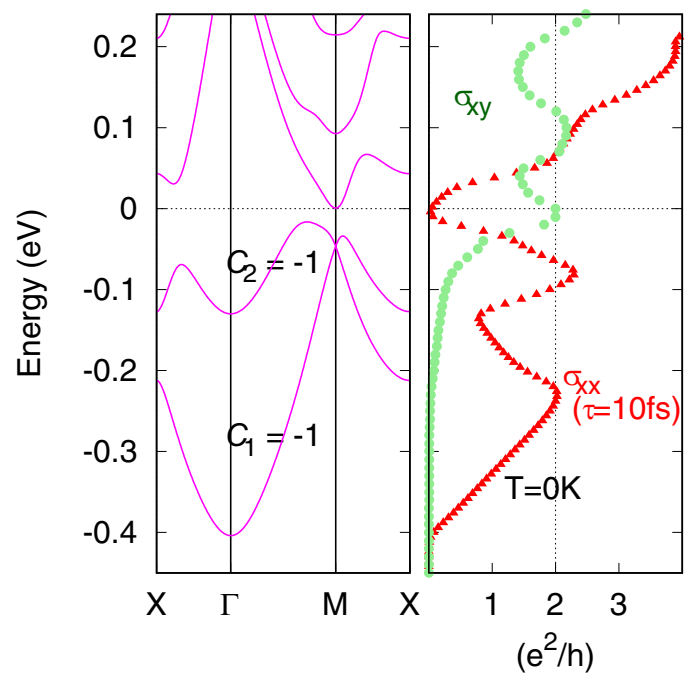

(c)

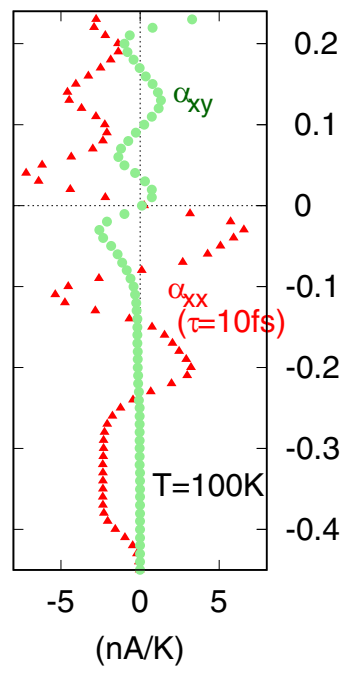

FIG. 5. (a) Zoomed-in band structure around the conduction band bottom, and the chemical potential dependence of (b) zero-temperature electric conductivities $\sigma_{x x}(\tau=10 \mathrm{fs})$ and $\sigma_{x y}$, and of (c) thermoelectric conductivities $\alpha_{x x}(\tau=10 \mathrm{fs})$ and $\alpha_{x y}$ at $100 \mathrm{~K}$. The system has two additional electrons as compared to the pristine system, which occupy the lowest two bands in (a), where their respective Chern numbers of -1 are indicated. A Gaussian smearing of $10 \mathrm{meV}$ is employed for (b). 